\title{
The Neural Network for Chemotaxis to Tastants in Caenorhabditis elegans Is Specialized for Temporal Differentiation
}

\author{
Tod R. Thiele, Serge Faumont, and Shawn R. Lockery \\ Institute of Neuroscience, University of Oregon, Eugene, Oregon 97403
}

\begin{abstract}
Chemotaxis in Caenorhabditis elegans depends critically on the rate of change of attractant concentration computed as the worm moves through its environment. This computation depends, in turn, on the neuron class ASE, a left-right pair of pair of chemosensory neurons that is functionally asymmetric such that the left neuron is an on-cell, whereas the right neuron is an off-cell. To determine whether this coding strategy is a general feature of chemosensation in C. elegans, we imaged calcium responses in all chemosensory neurons known or in a position to contribute to chemotaxis to tastants in this organism. This survey revealed one new class of on-cells (ADF) and one new class of off-cells (ASH). Thus, the ASE class is unique in having both an on-cell and an off-cell. We also found that the newly characterized on-cells and off-cells promote runs and turns, respectively, mirroring the pattern reported previously for ASEL and ASER. Our results suggest that the $C$. elegans chemotaxis network is specialized for the temporal differentiation of chemosensory inputs, as required for chemotaxis.
\end{abstract}

\section{Introduction}

Caenorhabditis elegans exhibits robust chemotaxis in concentration gradients of attractive odors and tastes. Chemotaxis results from two distinct behavioral mechanisms, both of which depend on the time derivative of attractant. The first mechanism is random walk that is biased by the time derivative of attractant concentration (Dusenbery, 1980; Pierce-Shimomura et al., 1999). When the derivative is negative, indicating movement down the gradient, the frequency of spontaneous reorienting turns rises, truncating movements in the wrong direction. When the derivative is positive, indicating movement up the gradient, the frequency of turns is suppressed, leading to long "runs" up the gradient. The second mechanism is klinotaxis, in which the normal sinusoidal movements of the worm during runs are biased in the direction of increasing concentration (Iino and Yoshida, 2009).

Chemical stimuli are sensed by three types of chemosensory organs. These include the amphids and the phasmids-left-right pairs of sensory organs in the head and tail, respectively — and the inner labial organs of the mouth, which have a sixfold symmetry. Only the amphids appear to be required for chemotaxis (Ward, 1973; Bargmann and Horvitz, 1991). Each amphid is innervated by three chemosensory neurons specialized for olfaction and

Received Feb. 3, 2009; revised Aug. 12, 2009; accepted Aug. 13, 2009.

This work was supported by the National Institute of Mental Health (Grant MH051383). We thank the Caenorhabditis Genetics Center and Samuel Laboratory for supplying worm strains. We thank Aristides Arrenberg for critical reading of this manuscript.

Correspondence should be addressed to Shawn R. Lockery, Institute of Neuroscience, University of Oregon, Eugene, 0R 97403. E-mail: shawn@uoregon.edu.

T. R. Thiele's present address: Department of Physiology, University of California, San Francisco, CA 94143. D01:10.1523/JNEUROSCI.0594-09.2009

Copyright $\odot 2009$ Society for Neuroscience $\quad$ 0270-6474/09/2911904-08\$15.00/0 eight chemosensory neurons specialized for taste. The taste neurons ASEL and ASER, collectively known by the class name ASE, play a dominant role in chemotaxis to the attractant $\mathrm{NaCl}$ and certain other inorganic salts (Bargmann and Horvitz, 1991; Pierce-Shimomura et al., 1999; Miller et al., 2005). Calcium imaging experiments show that ASEL and ASER are functionally asymmetric (Suzuki et al., 2008). ASEL is an on-cell in that it is transiently activated by increases in $\mathrm{NaCl}$ concentration, whereas ASER is an offcell in that it is transiently activated by decreases in $\mathrm{NaCl}$ concentration. The outputs of ASE neurons converge on the motor system but with opposing effects-activation of ASEL (the on-cell) promotes runs at the expense of turns, whereas activation of ASER (the off-cell) promotes turns at the expense of runs. Thus, at the point of convergence, the net effect of ASE activation is a behavioral signal that approximates the time derivative of attractant concentration.

It is not yet known whether on-off coding and left-right asymmetry are general features of the neuronal network for taste, as physiological recordings of chemosensory responses have been reported only for ASE and one other class of taste neurons in $C$. elegans (Hilliard et al., 2005; Suzuki et al., 2008). Here, we report the results of the first comprehensive survey of the physiological responses of taste neurons in C. elegans. We found two new neuron classes that exhibited calcium transients in response to stepwise changes in salt concentration, ADF and ASH. The two ADF neurons are on-cells, whereas the two ASH neurons are off-cells. Thus, on-cells and off-cells are a universal feature of sensory coding of tastants in C. elegans, but only the ASE neuron class contains both an off-cell and an on-cell. Using laser ablations combined with step response behavioral assays, we also found that newly identified on-cells and off-cells promote runs and turns, respectively, mirroring the pattern established previously for ASEL and ASER. Our results suggest that the C. elegans taste 
A

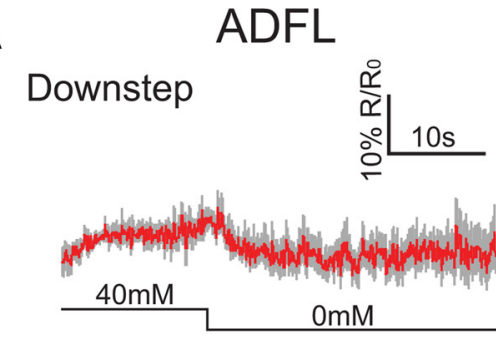

B Upstep

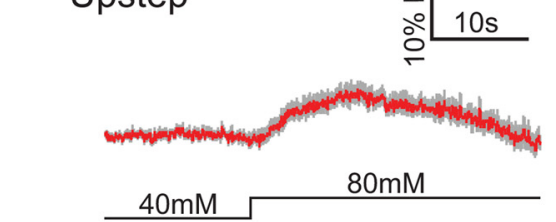

C

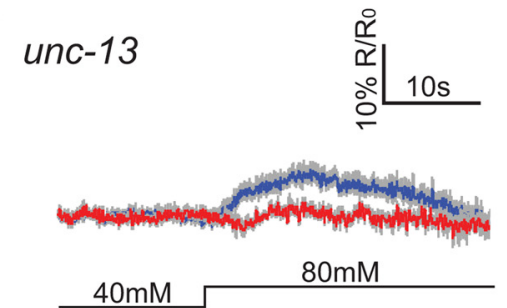

D

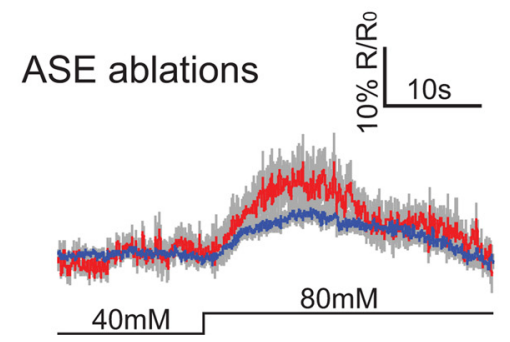

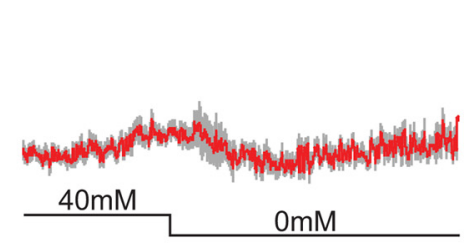

ADFR

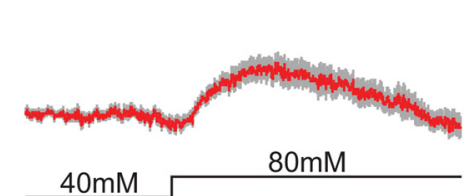

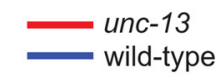
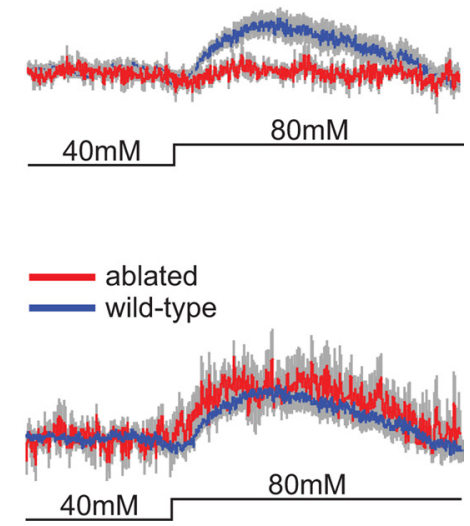

Figure 1. ADF neurons are on-cells. $\boldsymbol{A}, \boldsymbol{B}$, Average calcium transients in ADF neurons in wild-type animals in response to $\mathrm{NaCl}$ concentration steps of $\pm 40 \mathrm{~mm}$ from a baseline of $40 \mathrm{~mm}$. C, Average calcium transients in ADF recorded under similar conditions in unc-13 mutants. D, Average calcium transients in ADF recorded in wild-type animals in which the ASE neurons were ablated. In all panels, the concentration step is indicated below the calcium traces. The gray band represents $\pm S E M ; n=5$ recordings, with 1 recording per worm. The traces from $\boldsymbol{B}$ are shown in blue in $\boldsymbol{C}$ and $\boldsymbol{D}$ for comparison.

neurons, in conjunction with downstream elements of the C. elegans chemotaxis network, are specialized for the temporal differentiation of chemosensory inputs, the fundamental computation underlying this behavior.

\section{Materials and Methods}

Animals. The strains used in this study were wild-type C. elegans Bristol (N2), XL90 lin-15(n765); ntEx4[daf-7::YC3.60; lin-15(+)], XL117 lin-15(n765); ntEx16[ops-1::YC3.60, lin-15(+)], XL124 lin-15(n765); ntIs16[tph- 1::YC3.60; lin-15(+)], AQ1444 lin-15(n765); ljEx[sra-6::YC2.12; lin-15(+)] (Hilliard et al., 2005), XL132 unc-13(e51); ntIs16[tph- 1::YC3.60; lin-15(+)], XL131 unc13(e51); ljEx[sra-6::YC2.12; lin-15(+)], XL164 ntEx[gpa- 15::YC3.60], XL138 $n t E x 25[o d r-2 b:: Y C 3.60]$ and Ex[odr-4::YC3.60] (Gabel et al., 2007). Nematodes were grown in mixed-stage cultures at room temperature $\left(22-25^{\circ} \mathrm{C}\right)$ on $1.7 \%$ agar-filled plates containing nematode growth medium seeded with Escherichia coli strain OP50 (Brenner, 1974). All experiments were performed at room temperature $\left(22-25^{\circ} \mathrm{C}\right)$. Standard procedures were used to cross XL117 and AQ1444 with unc-13(e51).

Molecular biology. The tph-1::YC3.60 construct for ADF imaging was made by PCR amplification of the tph-1 promoter from genomic DNA and insertion into the HindIII and NotI sites of an expression vector containing the cameleon probe YC3.60 (Nagai et al., 2004). The expression vector is a modified pPD95.75 Fire Vector in which GFP (green

fluorescent protein) was replaced with YC3.60. The $t p h-1$ promoter contains $1.1 \mathrm{~kb}$ upstream of the ATG and the first three codons. This construct drives expression of YC3.60 exclusively in ADF. The ops-1::YC3.60 construct for ASG imaging was made by PCR amplification of the ops-1 promoter from genomic DNA and insertion into the HindIII and NotI sites of the YC3.60 expression vector. The ops-1 promoter contains $2 \mathrm{~kb}$ upstream of the ATG and drives expression exclusively in ASG. The daf-7::YC3.60 construct for ASI imaging was made by PCR amplification of the daf-7 promoter from genomic DNA and insertion into the NotI and NcoI sites of the YC3.60 expression vector. The daf-7 promoter contains $4.7 \mathrm{~kb}$ upstream of the ATG and drives expression in ASI and occasionally drives faint expression in two unidentified head neurons. The gpa-15::YC3.60 construct for ASK and ADL imaging was made by PCR amplification of the gpa-15 promoter from genomic DNA and insertion into the HindIII and NotI sites of the YC3.60 expression vector. The gpa-15 promoter contains $3 \mathrm{~kb}$ upstream of the ATG and drives expression in ASK, ADL, ASH, PHA, and PHB. All constructs were injected at 50 $\mathrm{ng} / \mu \mathrm{g}$ using standard methods (Mello and Fire, 1995).

Calcium imaging. Young adult animals were glued to a $1.5 \%$ agarose pad (30 mM TAPS [ $N$-tris(hydroxymethyl)methyl-3-aminopropanesulphonic acid], $\mathrm{pH}$ 9) using cyanoacrylate glue (Nexaband S/C; Abbott Laboratories); animals were briefly cooled during the gluing process. A two-channel, gravity-fed perfusion pencil (360 $\mu \mathrm{m}$ tip), whose flow was controlled by programmable upstream valves (AutoMate Scientific), was placed in close proximity $(\sim 0.5$ $\mathrm{mm}$ ) to the nose of each animal. The flow rate was $0.3 \mathrm{ml} / \mathrm{min}$ and the total bath volume was $\sim 0.2 \mathrm{ml}$. Local perfusion, and thus rapid solution exchange $\left(t_{1 / 2}=0.5 \mathrm{~s}\right)$, was achieved by locating the worm in a fluid plume between the inflow perfusion pencil and the outflow pipette. Solutions contained the indicated amount of $\mathrm{NaCl}$ plus the following (in mM): 10 HEPES-NaOH, pH 7.1, 1 $\mathrm{CaCl}_{2}, 1 \mathrm{MgSO}_{4}$; osmolarity was adjusted to $350 \mathrm{mOsm}$ with glycerol. Optical recordings were performed on a Zeiss Axiovert 135, using a Zeiss Plan-Apochromat $63 \times$ oil, 1.4 numerical aperture objective. The microscope was fitted with a Hamamatsu ORCA AG CCD camera (Hamamatsu Photonics), an Optical Insights Dual-View beam splitter (Photometrics), and an electronic shutter (ASI). The genetically encoded calcium probe cameleon was used in all experiments. Images were acquired at 10-20 Hz using MetaVue software (version 6.2r2; Molecular Devices). Image stacks were analyzed using the Jamlyze program, as previously described (Kerr et al., 2000). The yellow fluorescent protein (YFP)/cyan fluorescent protein (CFP) emission ratio was computed as follows: (YFP intensity)/(CFP intensity) -0.65 , where the latter term corrects for CFP bleedthrough into the YFP channel. Photobleaching was corrected by fitting a single exponential to inactive portions of the ratio trace and dividing the latter by the fitted curve.

Neuronal ablations. Cells were killed using a laser as described previously (Bargmann and Avery, 1995). L1 animals (N2) were mounted on 2.5\% agarose pads containing 5-7 mM immobilizing agent $\mathrm{NaN}_{3}$. ASE neurons were identified by position. Animals were remounted $1-3 \mathrm{~h}$ after surgery to confirm the ablation; those exhibiting collateral damage were discarded. Shamoperated animals were treated in the same manner except that the laser was not fired. Step response behavior was tested $3 \mathrm{~d}$ after ablation. 
Step response assay. The step response apparatus has been described previously (Miller et al., 2005). Worms were placed individually on a thin porous membrane supported over a yoked pair of miniature inverted showerheads. Each showerhead emitted a saline solution with a different salt concentration. Stepwise concentration changes were delivered by sliding the showerhead assembly relative to the worm. The behavior of individual worms was scored manually by pressing computer keys to record changes in behavioral state. Ablated and sham animals were tested in an alternating sequence, with the experimenter blind to animal condition. Solutions used in the assay were identical with solutions used during $\mathrm{NaCl}$ imaging experiments.

Behavioral statistics. The forward probabilities $(p)$ for step response experiments were transformed as $2 \arcsin (p)$ to compensate for the compression of variance at extremes of the probability scale (Winer et al., 1991); this transformation is conservative because it increases the variance at the extremes. Statistical significance was assessed by a two-factor repeated-measures ANOVA applied to forward probabilities during a poststimulus analysis window. In addition, we made planned pairwise comparisons ( $t$ tests) on mean behavioral-state probabilities at each time point in the analysis window. The significance level of each $t$ test was adjusted using the Bonferroni correction for multiple comparisons.

\section{Results}

To determine whether the coding strategy exhibited by amphid chemosensory neuron class ASE is a general feature of chemosensory neurons in $C$. elegans, we studied the function of each of the other classes of chemosensory neurons that are directly exposed to the fluid surrounding the worm or contained in its mouth. These included the seven other classes of exposed amphid neurons, two classes of phasmid neurons, and one class of neurons in the inner labial sensillae. Given the left-right symmetry of the amphid and phasmid organs, and the sixfold symmetry of the inner labial sensillae, this experimental plan dictated multiple recordings from each of 24 distinct types of neurons. Thus, to keep the number of recordings manageable, we used changes in $\mathrm{NaCl}$ concentration as a representative stimulus for the class of soluble ionic attractants to which ASE neurons are known to respond ( $\mathrm{Su}-$ zuki et al., 2008).

\section{Amphid chemosensory neurons}

$A D F$

Previous laser ablation studies have shown that killing the amphid neuron class ADF in conjunction with ASG and ASI causes a deficit in $\mathrm{NaCl}$ chemotaxis, whereas killing ADF alone has no effect, suggesting it acts weakly and redundantly with ASI and ASG (Bargmann and Horvitz, 1991). To monitor activity in ADF, we expressed cameleon YC3.6 under the control of the
ASIL

ASIR

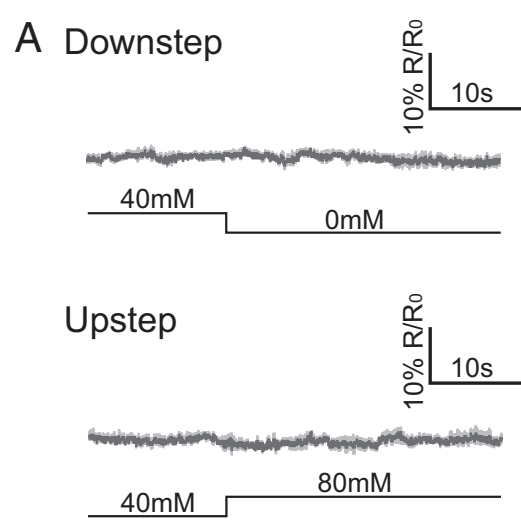

ASGL
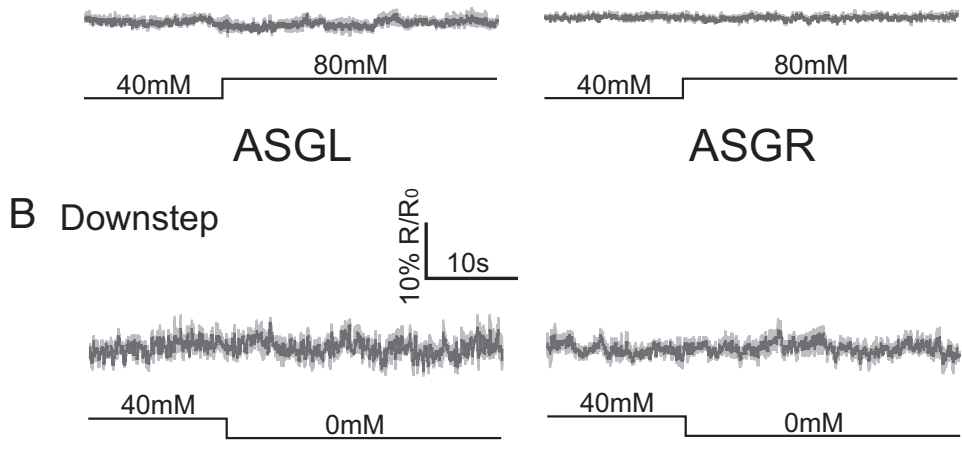

ASGR
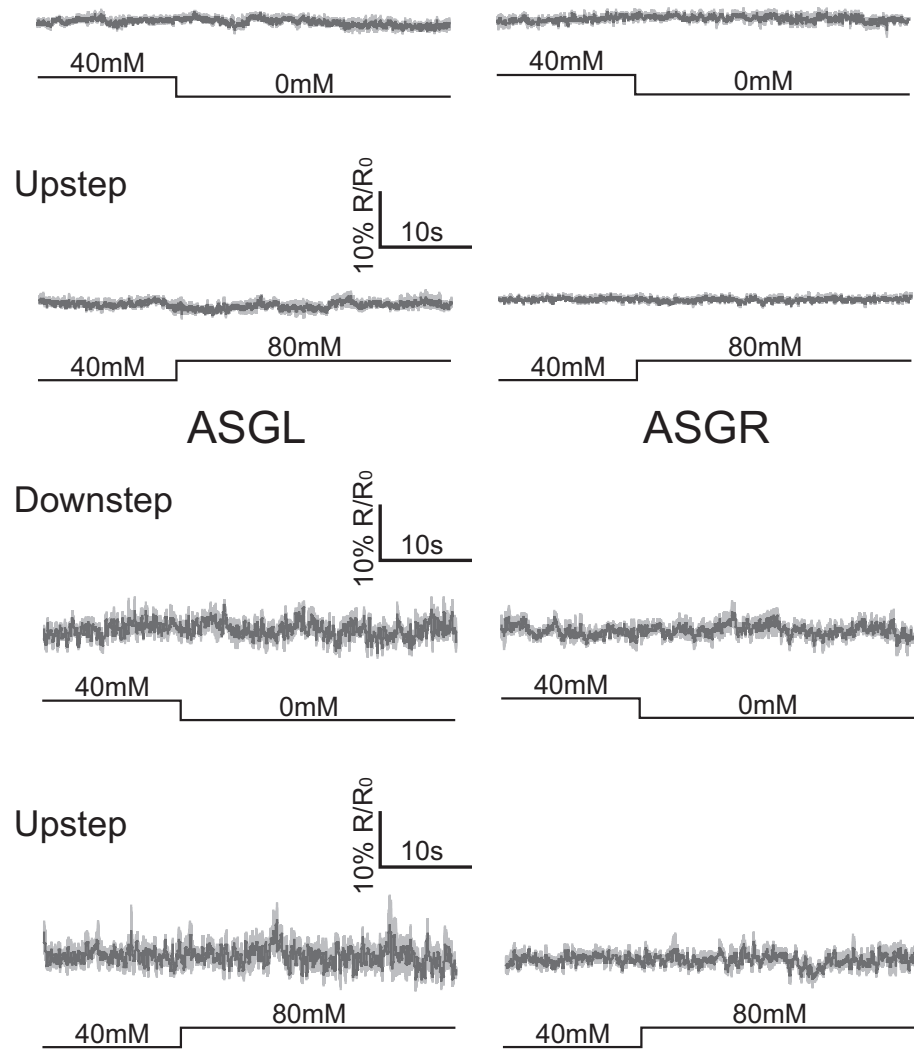

ASI
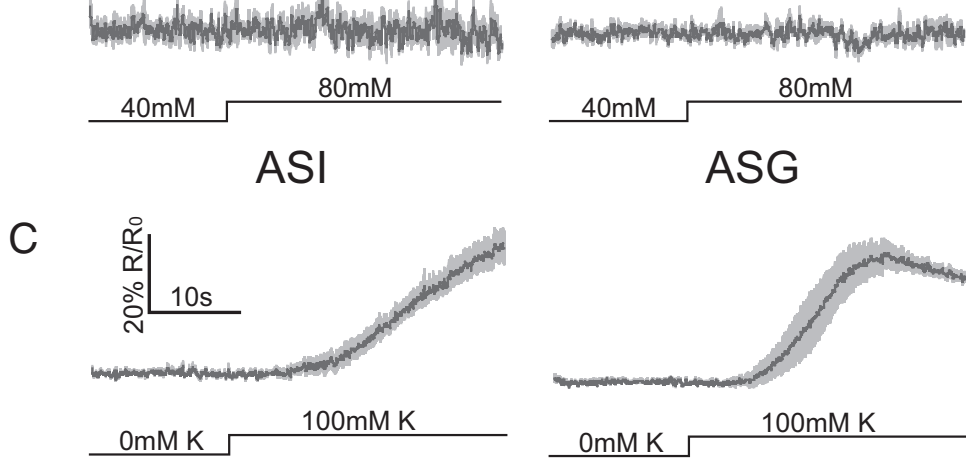

ASG

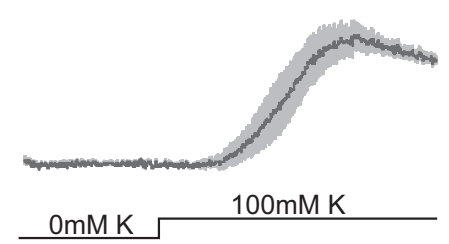

Figure 2. ASI and ASG neurons are unresponsive to $\mathrm{NaCl}$ concentration steps. $\boldsymbol{A}, \boldsymbol{B}$, Average calcium transients in ASI and ASG neurons in wild-type animals in response to $\mathrm{NaCl}$ concentration steps of $\pm 40 \mathrm{~mm}$ from a baseline of $40 \mathrm{~mm}$. The concentration step is indicated below the calcium traces. The gray band represents $\pm S E M ; n=5$ recordings, with 1 recording per worm. C, Direct stimulation of ASI and ASG via high $\mathrm{K}^{+}$saline delivered through an incision in the cuticle; $n=4$, with 1 recording per worm.

tph-1 promoter which drives expression in this neuron class (Sze et al., 2000). Both the left and right ADF neurons (ADFL and ADFR) exhibited an increase in intracellular calcium in response to $\mathrm{NaCl}$ upsteps and a small decrease intracellular calcium in response to $\mathrm{NaCl}$ downsteps (Fig. 1A,B). Thus, ADF is functionally symmetric across the midline and acts as an on-cell in the sense that its preferred stimulus is an increase in $\mathrm{NaCl}$.

To test whether $\mathrm{NaCl}$ responses in $\mathrm{ADF}$ are direct or synaptically mediated, we recorded upstep and downstep responses in ADF in unc-13(e51) mutants, which have a profound deficit in synaptic vesicle exocytosis (Richmond et al., 1999). We found that $\mathrm{ADF}$ responses to upsteps and downsteps were eliminated in unc-13 mutants (Fig. 1C) (data not shown), suggesting that these responses are synaptic in origin. To test whether ASE neurons provide this synaptic input, we monitored $\mathrm{NaCl}$ responses of 


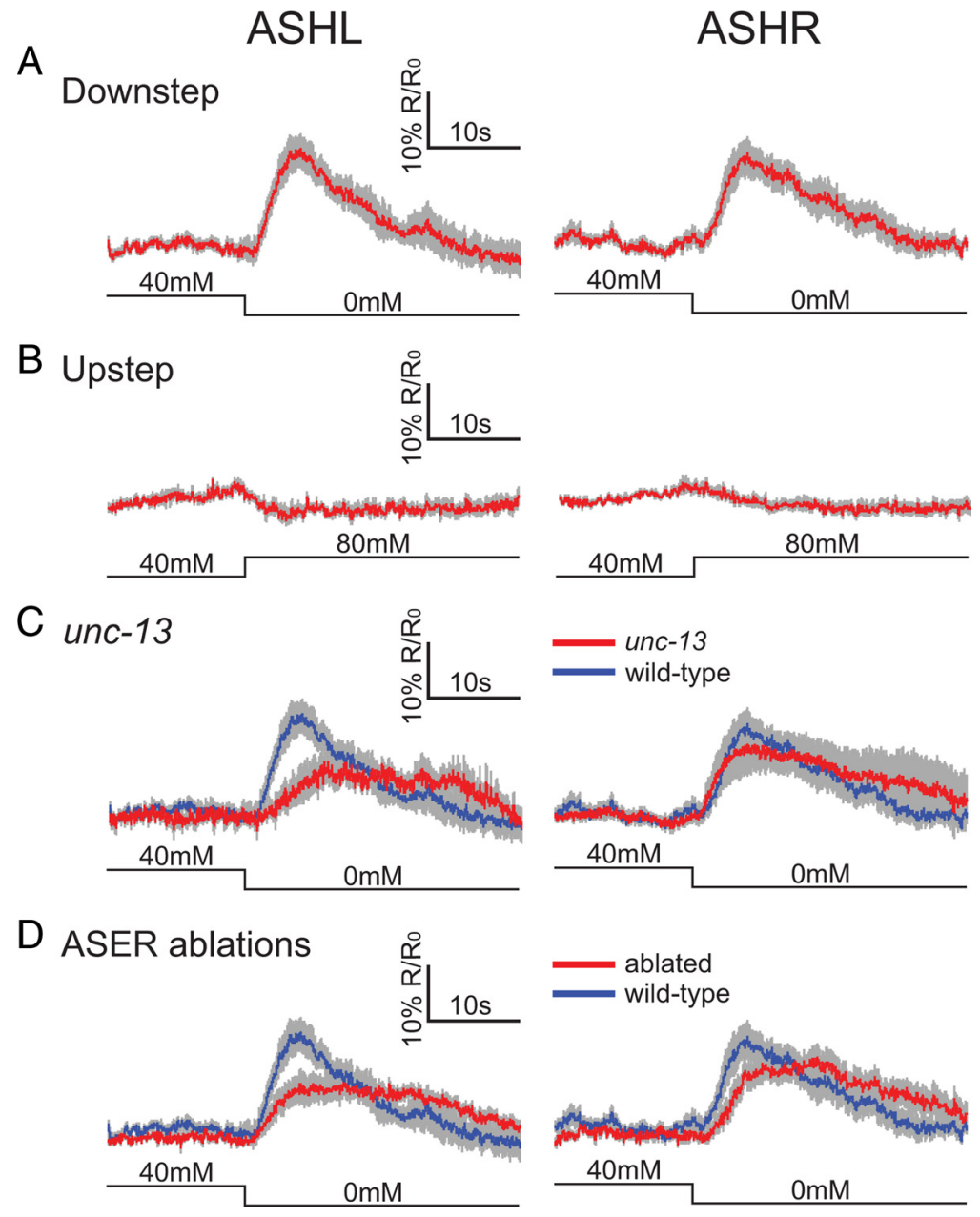

Figure 3. ASH neurons are off-cells. $A, B$, Average calcium transients in ASH neurons in wild-type animals in response to $\mathrm{NaCl}$ concentration steps of $\pm 40 \mathrm{~mm}$ from a baseline of $40 \mathrm{~mm}$. C, Average calcium transients in ASH recorded under similar conditions in unc-13 mutants. D, Average calcium transients in ASH recorded in wild-type animals in which ASER was ablated. In all panels, the concentration step is indicated below the calcium traces. The gray band represents \pm SEM; $n=5$ recordings, with 1 recording per worm. The traces from $\boldsymbol{A}$ are shown in blue in $\boldsymbol{C}$ and $\boldsymbol{D}$ for comparison.

ADF neurons in animals in which ASEL and ASER had been ablated. We found that $\mathrm{ADF}$ responses were essentially normal in the ablated animals (Fig. 1D), arguing against ASE as the source of sensory input to ADF. Thus, ADF appears to be postsynaptic to one or more $\mathrm{NaCl}$-sensitive neurons other than the ASE neurons; alternatively, synaptic transmission could somehow be required for normal development of intrinsic $\mathrm{NaCl}$ sensitivity in $\mathrm{ADF}$.

\section{$A S G$ and $A S I$}

The amphid neuron classes ASG and ASI act weakly and redundantly with $\mathrm{ADF}$ during $\mathrm{NaCl}$ chemotaxis (Bargmann and Horvitz, 1991). We imaged ASI and ASG using strains in which cameleon was expressed under the control of the daf-7 and ops-1 promoters, respectively (Schackwitz et al., 1996; Sarafi-Reinach et al., 2001). Surprisingly, neither ASI nor ASG exhibited calcium transients either to $\mathrm{NaCl}$ upsteps or downsteps (Fig. 2A,B). To ensure that the cameleon protein was functional in these strains, we partially dissected several animals and depolarized ASI and ASG directly using a high potassium saline. Large ratio increases were observed, indicating that the cameleon protein was functional in both strains (Fig. 2C). The absence of detectable calcium transients in ASI and ASG conflicts with the results of the previous ablations described above (Bargmann and Horvitz, 1991).
One possibility, consistent with the weak effects of ASI and ASG ablations, is that these neurons do respond to $\mathrm{NaCl}$, but their calcium transients are small and below the threshold for detection by the cameleon protein. Another possibility is that ASI and ASG do not respond to $\mathrm{NaCl}$ but they are required for the development or function of other neurons.

\section{ASH}

The amphid neuron class ASH is sensitive to a variety of noxious stimuli including chemical repellents, osmotic shock, and nose touch (Hilliard et al., 2005). Consistent with its role in detecting noxious stimuli, ASH resembles ASER in that it elicits avoidance responses when stimulated (Tobin et al., 2002).

To monitor activity in ASH, we used a previously published cameleon strain in which cameleon is expressed under the control of the sra- 6 promoter (Hilliard et al., 2005). Both the left and right ASH neurons exhibited a large increase in intracellular calcium in response to $\mathrm{NaCl}$ downsteps and a small decrease in intracellular calcium in response to $\mathrm{NaCl}$ upsteps (Fig. 3A,B). Thus, ASH is functionally symmetric across the midline and acts as an off-cell in the sense that its preferred stimulus is a decrease in $\mathrm{NaCl}$. Our results differ from a previous study that did not find $\mathrm{NaCl}$ sensitivity in ASH (Hilliard et al., 2005). One possible explanation is that the other study focused on responses to $\mathrm{NaCl}$ upsteps, which we show here produce a relatively modest decrease in the calcium signal that may not have been apparent under the conditions of the previous experiments.

To test whether $\mathrm{NaCl}$ responses in ASH are direct or synaptically mediated, we recorded upstep and downstep responses in ASH in unc-13(e51) mutants (Fig. 3C). We found that calcium transients in ASHR were essentially normal, indicating that $\mathrm{NaCl}$ responses in this neuron may be direct. In ASHL, however, calcium transients were present but reduced in amplitude. This result suggests that, whereas $\mathrm{NaCl}$ responses in ASHR are direct, $\mathrm{NaCl}$ responses in ASHL may be the sum of direct and indirect inputs. The demonstration that ASH neurons respond directly to decreases in salt concentration adds a new modality to the repertoire of this polymodal neuron. ASH has previously been shown to be required for avoidance responses to a wide range of stimuli that includes nose touch (Kaplan and Horvitz, 1993), bitter, toxic, and noxious chemicals (Hart et al., 1999; Sambongi et al., 1999; Hilliard et al., 2005), low pH (Sambongi et al., 2000), and high osmolarity (Hart et al., 1999).

A likely candidate for synaptic input to ASHL is ASER which, like ASHL, is an off-cell. We therefore recorded $\mathrm{NaCl}$ responses in ASH neurons in animals in which ASER was ablated. We found that ASHL responses in ablated animals were reduced in amplitude by an amount that closely matched the reduction seen in unc-13 synaptic mutants (Fig. 3D). We conclude that some or all of the synaptic input to ASHL during $\mathrm{NaCl}$ downsteps comes 
from ASER. The connection from ASER to ASHL is likely to be polysynaptic because there is no anatomical evidence for monosynaptic chemical or electrical connections between ASER and ASHL (White et al., 1986). It is presently unclear why ASER sends input only to the contralateral ASH neuron. This asymmetry could be an anomaly or the result of unknown functional differences between ASH neurons. We note, however, that this asymmetry is confined to the source of inputs and does not affect the fact that both ASH neurons are off-cells. We did not test ASEL for synaptic effects on ASH because ASEL does not appear to respond to downsteps (Suzuki et al., 2008).

Our finding that ASH responds to $\mathrm{NaCl}$ raised the question of whether ASH activity is responsible for the synaptic input underlying $\mathrm{NaCl}$ responses in ADF. To test this possibility, we imaged ADF in animals in which both ASH and ASE had been ablated, in case these neuron classes might act jointly to excite ADF during $\mathrm{NaCl}$ upsteps. However, we found that ADF responses were preserved in animals lacking both ASH and ASE, ruling out ASH as the synaptic source for the onresponses we observe in ADF (supplemental Fig. 1, available at www.jneurosci. org as supplemental material).

\section{$A S J, A S K$, and $A D L$}

The amphid neuron classes ASJ, ASK, and ADL have been implicated in a variety of chemosensory behaviors including minor roles in chemotaxis (ASJ, ASK) and avoidance (ADL) (Bargmann and Horvitz, 1991; Troemel et al., 1995). We imaged these neurons in strains in which cameleon was expressed under the control of the promoters odr-4 (ASJ), gpa-15 (ASK), and gpa-15 (ADL). None of these neuron classes exhibited calcium transients either to $\mathrm{NaCl}$ upsteps or downsteps (supplemental Fig. 2, available at www.jneurosci.org as supplemental material).

\section{Phasmid chemosensory neurons}

The neuron classes PHA and PHB innervate the phasmid chemosensory organs of the tail. These neurons are required for chemosensory avoidance responses initiated when the tail encounters a chemical repellent (Hilliard et al., 2002). We imaged these neurons in strains in which cameleon was expressed under the control of the odr-4 promoter. Neither PHA nor PHB exhibited calcium transients to $\mathrm{NaCl}$ upsteps or downsteps (supplemental Fig. 3, available at www.jneurosci.org as supplemental material).

\section{Inner labial sensory neurons}

The neuron classes IL1 and IL2 innervate the six inner labial sensillae located in the worm's mouth. We imaged IL2 neurons in
ADF ablations
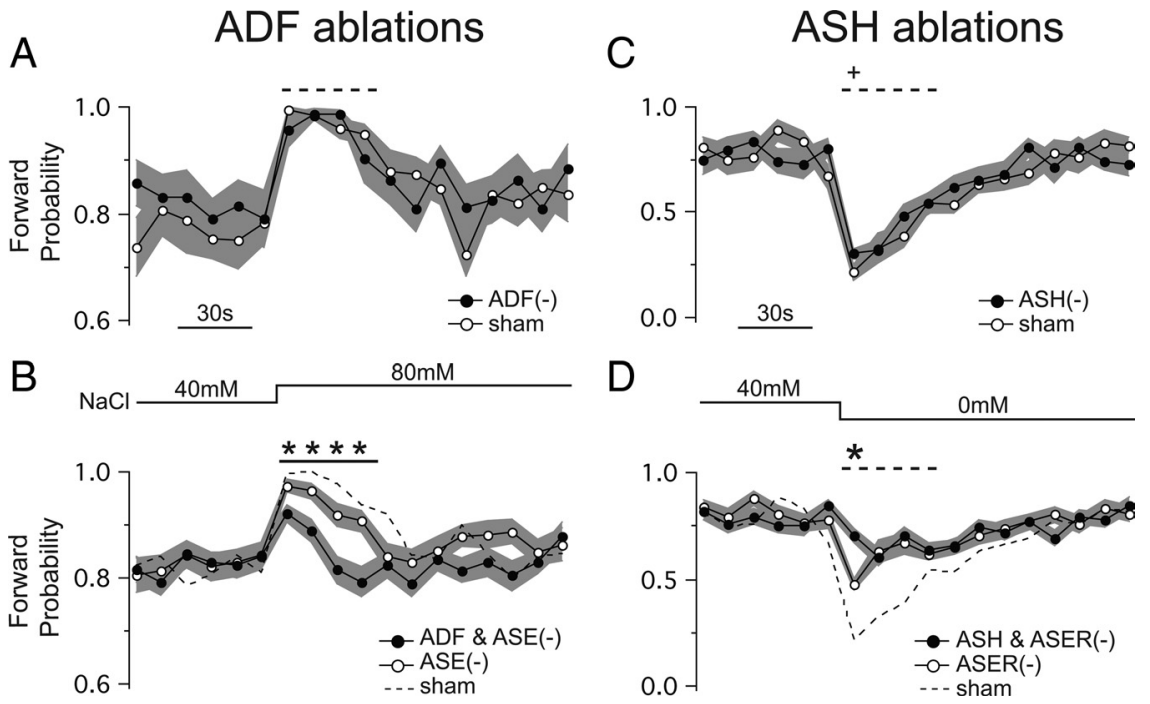

Figure 4. Behavioral contributions of ADF and ASH responses to preferred stimuli. Probability of forward locomotion is plotted against time relative to the concentration step. $\boldsymbol{A}$, Run behavior in animals lacking ADF neurons. $\boldsymbol{B}$, Run behavior in animals lacking DF and ASE neurons. C, Turn behavior in animals lacking ASH neurons. D, Turn behavior in animals lacking ASH and ASER neurons. ANOVA over the time windows indicted by the horizontal lines above the traces. Solid lines, Significance at $p<0.05$; dashed lines, significant. The asterisks indicate significant differences between control and experimental means ( $t$ test, $p<0.05$ after Bonferroni correction); the pluses indicate significance between means in uncorrected comparisons. The gray band represents \pm SEM with $n=15$ except for $C$ in which $n=13$.
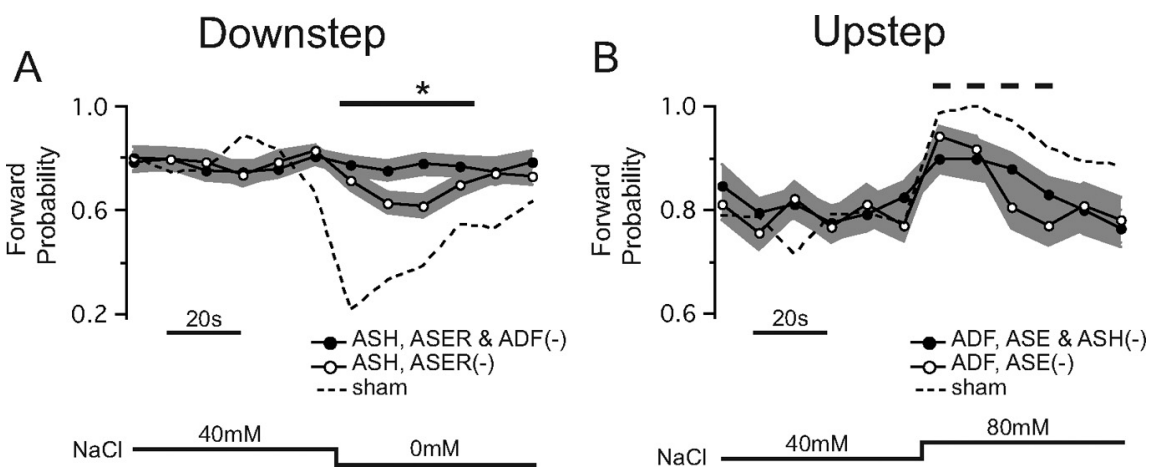

Figure 5. Behavioral contributions of ADF and ASH responses to nonpreferred stimuli. Probability of forward locomotion is plotted against time relative to the concentration step. $\boldsymbol{A}$, Turn behavior in animals lacking ASER, ASH, and ADF. $\boldsymbol{B}$, Run behavior in animals lacking ADF, ASE, and ASH. The concentration step is indicated between the probability traces. Statistical significance was assessed via a repeated-measures ANOVA over the indicated time window (horizontal lines above traces) after the step. Solid lines, Significance at $p<0.05$; dashed lines, not significant. The asterisks indicate significant differences between control and experimental means ( $t$ test, $p<0.05$ after Bonferroni correction); the pluses indicate significance between means in uncorrected comparisons. The gray band represents \pm SEM with $n=20$ in each panel.

strains in which cameleon was expressed under the control of the $o d r-2 b$ promoter. IL2 did not respond to $\mathrm{NaCl}$ upsteps or downsteps (supplemental Fig. 3, available at www.jneurosci.org as supplemental material). IL1, which is primarily a mechanosensory neuron (Hart et al., 1995), was excluded from our survey.

\section{Behavioral effects of responses to preferred stimuli}

Having identified the subset of chemosensory neurons that are activated or deactivated by changes in $\mathrm{NaCl}$ concentration, we next sought to determine the behavioral effects of neurons in this subset. This was done by ablating candidate neurons and testing for changes in the turn or run behaviors elicited by $\mathrm{NaCl}$ upsteps and downsteps, respectively (Miller et al., 2005; Suzuki et al., 2008). 


\section{$A D F$}

The preferred stimulus of ADF is an upstep in $\mathrm{NaCl}$, which elicits run behavior. To test whether ADF contributes to runs, we assayed run behavior in animals in which both ADF neurons had been ablated. We found that run behavior in ablated animals was statistically indistinguishable from that of sham-operated controls tested in parallel (Fig. $4 A)\left(F_{(1,33)}=0.07 ; p=0.21\right)$. This result could mean that ADF does not contribute to runs. However, it is also possible that the contribution of ADF is masked by large, behaviorally saturating effects of other neurons acting in parallel. To unmask a possible contribution from ADF, we tested the effects of ADF ablations in animals in which ASE neurons were also ablated. ASE neurons are likely candidates for parallel effects because ablation of either ASEL or ASER reduces run behavior, the normal response to upsteps (Suzuki et al., 2008). Indeed, we found that ablating ADF and ASE caused a significantly greater run deficit than ablating ASE alone (Fig. $4 B)\left(F_{(1,89)}=\right.$ $22.2 ; p<0.001)$. We conclude that, in response to its preferred stimulus, ADF acts redundantly with ASE neurons to elicit runs.

ASH

The preferred stimulus of $\mathrm{ASH}$ is a downstep in $\mathrm{NaCl}$, which elicits turns. To determine whether ASH contributes to turning, we tested animals in which both ASH neurons were ablated. We found that turning behavior in ablated animals was statistically indistinguishable from that of sham-operated controls (Fig. 4C) $\left(F_{(1,24)}=1.9 ; p=0.18\right)$. One interpretation of this result is that ASH does not contribute to turns induced by decreasing salt. This interpretation is consistent with the finding that killing $\mathrm{ASH}$, alone, does not impair chemotaxis tested in a shallow gradient assay (Bargmann and Horvitz, 1991) with the caveat that the rates of concentration change experienced by worms in shallow salt gradients are several orders of magnitude smaller than the rates experienced in the present study. However, it is possible that ASH acts redundantly with other neurons, as does ADF (Fig. 4B). Indeed, we found that ablating ASH jointly with ASER caused a significantly greater turn deficit than ablating ASER alone (Fig. $4 D)\left(t_{(42)}=4.1 ; p<0.001\right)$. We conclude that, in response to its preferred stimulus, ASH acts redundantly with ASER to cause turns.

\section{Behavioral effects of responses to nonpreferred stimuli $A D F$}

The persistence of turn behavior in response to downsteps in animals lacking ASH and ASER (Fig. 4D) suggests that there is at least one other sensory neuron that contributes to turns. According to the imaging data presented above (Figs. 1-3), the only other sensory neurons that respond during downsteps are the ADF neurons, which are deactivated by this stimulus. Because ADF activation causes runs (Fig. $4 B$ ), its deactivation could contribute to turns by suppressing runs, provided, of course, that ADF is tonically active. If so, then ablating ADF in animals lacking ASH and ASER would reduce turning behavior relative to animals lacking only ASH and ASER. We found that this was indeed the case (Fig. $5 A)\left(F_{(1,35)}=7.2 ; p<0.05\right)$. Moreover, turning appeared to be completely eliminated. We draw two main conclusions from this experiment. First, ASH, ASER, and $\mathrm{ADF}$ together mediate all of the sensory input required for turning, with the proviso that sensory responses in ADF are likely to be synaptic in origin. Second, ADF neurons are tonically active. Similar findings have been reported for ASER and AWC chemosensory neurons (Chalasani et al., 2007; Suzuki et al., 2008).
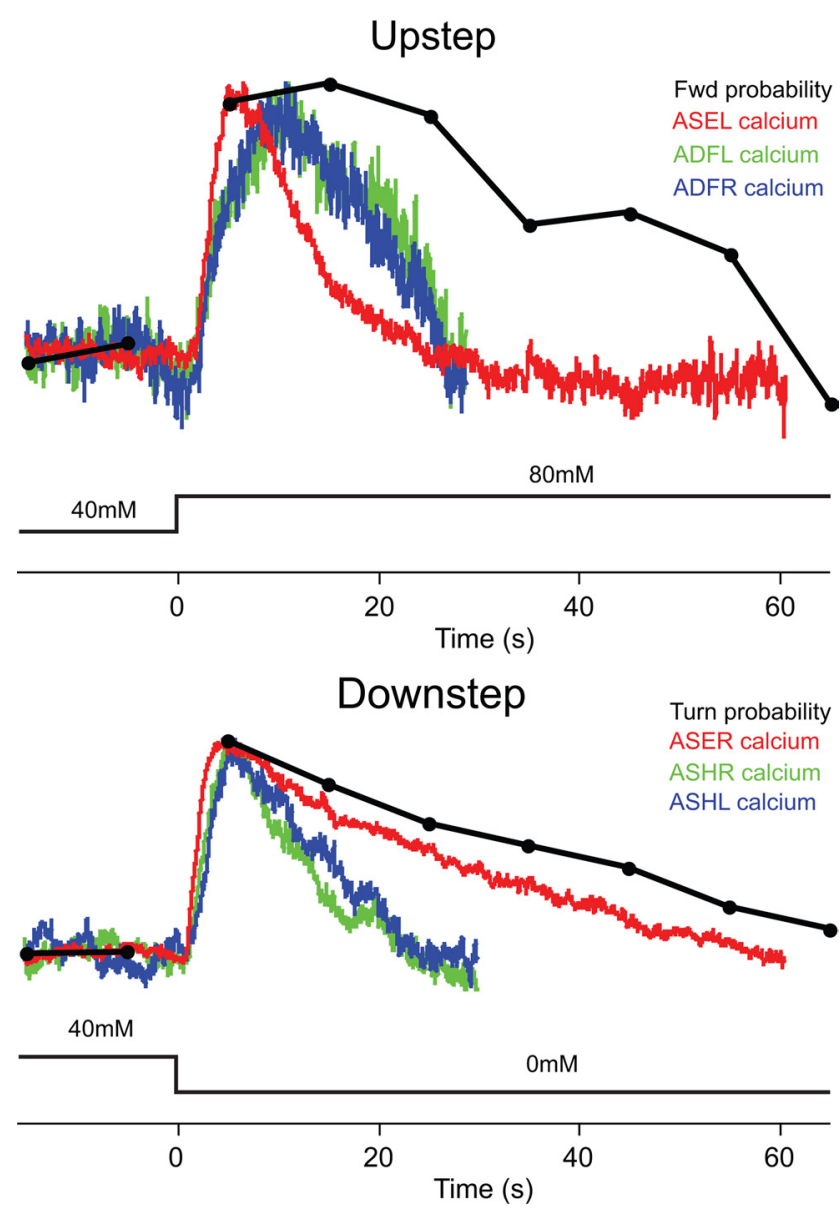

Figure 6. Temporal relationship between behavioral probabilities and the calcium transients in chemosensory neurons for upsteps and downsteps. In each panel, the behavioral probability and calcium traces were scaled such that the amplitude of the change from baseline is the same. The concentration step is indicated below the traces. ASE calcium transients are from the study by Suzuki et al. (2008). The sham experiments from Figure 4 and Suzuki et al. (2008), their supplemental Figure 6, were used to generate the behavioral traces.

ASH

The persistence of residual run behavior in response to upsteps in animals lacking ADF and ASE (Fig. $4 B$ ) suggests that there is at least one other sensory neuron contributing to runs. The only other sensory neurons known to respond to upsteps are the ASH cells, which are deactivated by this stimulus. Applying logic similar to that used in the case of ADF, ASH deactivation could contribute to runs. If so, then ablating ASH in animals lacking all $\mathrm{ADF}$ and ASE neurons would reduce run behavior relative to animals lacking only ADF and ASE. However, we found no additional effect of ablating ASH (Fig. $5 B)\left(F_{(1,46)}=1.1 ; p=0.29\right)$. Thus, the deactivation seen in ASH in response to its nonpreferred stimulus does not have a detectable effect on behavior. Furthermore, ASH, ASE, and ADF neurons do not mediate all of the sensory input required for normal levels of run behavior; another pathway remains to be found.

\section{Regulation of behavioral dynamics}

The final common path for the regulation of the direction of locomotion in C. elegans is a small network of premotor command interneurons (Chalfie et al., 1985; Zheng et al., 1999). Inspection of the C. elegans anatomical wiring diagram reveals that neuronal pathways from the taste chemosensory neurons to the 
command interneurons are overwhelmingly polysynaptic. This observation raised the question of whether the time course of behavioral responses is set by sensory neurons or by interneurons, including those in the polysynaptic pathways as well as the command interneurons themselves. To address this question, we compared the average time course of calcium transients in chemosensory neurons with the average time course of changes in the probability of forward locomotion induced by $\mathrm{NaCl}$ upsteps and downsteps (Fig. 6). Overall, the relative latencies, of calcium transients and behavioral responses were consistent with the former causing the latter.

Additional comparisons provided additional insights into sensory processing. In the case of upsteps, behavior decayed much more slowly than the calcium transients in any of the on-cells. One interpretation of this result is that the duration of the behavioral response is determined primarily by a sensory neuron that remains to be identified. A second interpretation is that the interneurons in the polysynaptic pathways act to prolong the behavioral responses, possibly by integrating sensory input. In the case of downsteps, behavior decayed more slowly than calcium transients in ASH, but at essentially the same rate as the calcium transient in ASER. This result, together with the fact that the behavioral effect of ASH is weak and redundant with ASER (Fig. 4), suggests a model in which the time course of the downstep behavioral responses is set primarily by ASER. We note that behavioral responses that decay more slowly than calcium transients in sensory neurons have been reported in the case of oxygen-sensing neurons in C. elegans (Zimmer et al., 2009).

\section{Discussion}

We have shown that changes in $\mathrm{NaCl}$ concentration are represented in distributed manner by an array of three neuron classes: ASE (Suzuki et al., 2008), and now ADF and ASH (Fig. 7). Like the ASE class, ADF and ASH have a preferred stimulus direction-ADF neurons are on-cells, whereas ASH neurons are offcells. Thus, on-off coding is a general feature of C. elegans chemosensory neurons involved in taste, at least for the size of concentration steps used here. In contrast, the left-right functional asymmetry of ASE neurons does not extend to ADF and $\mathrm{ASH}$, as each of these classes is symmetric with respect to being on-cells or off-cells. Anatomical reconstructions indicate that ADFL and ADFR are connected by gap junctions, as are ASHL and ASHR; such connections may help to ensure functional symmetry within these classes. Consistent with this view, the functionally asymmetric ASE neurons are not connected by gap junctions.

Our delineation of the taste network remains incomplete in two respects. First, we cannot yet account for the $\mathrm{NaCl}$ responses of ADF, as neither ASE nor ASH appear to provide this synaptic input (Fig. 1D). Second, we cannot yet account for a significant fraction of the run behavior triggered by $\mathrm{NaCl}$ upsteps, as upstep runs survive ablation of all chemosensory neurons known to respond to upsteps (Fig. 5B). In perhaps the simplest model to account for the missing sensory and behavioral functions, a single chemosensory neuron (or neuron class) remains to be identified, as indicated in Figure 6 by neuron X. This class is predicted to contain one or more on-cells that excite ADF, or off-cells that tonically inhibit ADF. Furthermore, this class is predicted to contribute to an output pathway that is independent of ADF, ASE, and ASH (Fig. 6). In light of previous ablation studies, ASI and ASG are the most likely candidates, although anatomical connections from these neurons to ADF are indirect (White et al., 1986). Identification of the missing neurons may require novel calcium probes with higher sensitivities or more favorable dynamic ranges than the one used here, or the use of patch-clamp electrophysiological approaches that remain to be developed for chemosensory studies.

The chemosensory neurons do not in themselves represent the derivative of the time course of concentration. For example, the derivative of a finite concentration step is a positive impulse at the onset of the step followed by a negative impulse at the offset of the step (Fig. 6). Although the calcium transients of the chemosensory neurons can be considered as approximations of these impulses, the chemosensory neurons represent the derivative incompletely, in two key respects. First, the on-cells (ASEL and ADF) exhibit only positive impulses, whereas the off-cells (ASER and ASH) exhibit only negative impulses. Second, the off-cell impulse is inverted. Nevertheless, previous studies have shown that the complete derivative is represented at the behavioral level (Miller et al., 2005) in that the probability of the orientation behaviors in response to concentration steps exhibits positive and negative impulses at the onset and offset of the step, respectively. That fact that the derivative is present at the behavioral level implies that the responses of on-cells and off-cells are integrated by the nervous system.

The present findings, in combination with previous results (Suzuki et al., 2008), suggest that this integration occurs at the network level. The final common path for the regulation of the direction of locomotion in C. elegans is a small network of pre- 
motor command interneurons (Chalfie et al., 1985; Zheng et al., 1999). Ablation experiments (Figs. 4, 5), together with direct activation of chemosensory neurons (Tobin et al., 2002; Suzuki et al., 2008), show that chemosensory information from both the on-cells and the off-cells converges at or before the command neurons. Thus, the command neurons, or interneurons upstream of them, assemble the derivative from distinct components provided by the chemosensory neurons. Importantly, the overall polarity of the synaptic pathways that mediate this convergence are such that the on-cell impulses retain their positive sign, whereas the off-cell impulses are inverted. In this sense, the $C$. elegans chemotaxis network, defined as the set of sensory neurons and interneurons directly involved in regulation of locomotion by chemoattractants, is specialized for temporal differentiation, the fundamental computation for chemotaxis in C. elegans (Pierce-Shimomura et al., 1999).

The mechanism of a biased random walk is known to contribute to three commonly studied spatial orientation behaviors in C. elegans: chemotaxis, aerotaxis, and thermotaxis (PierceShimomura et al., 1999; Ryu and Samuel, 2002; Zariwala et al., 2003; Chalasani et al., 2007; Zimmer et al., 2009). In each behavior, the complete derivative is represented in the probability or frequency of specific orientation responses. This commonality allows for meaningful comparisons of the neural circuits underlying these behaviors. In the two cases of orientation to chemicals (chemotaxis and aerotaxis), the derivative appears to be computed partly at the cellular level and partly at the network level as the main sensory neurons are either on-cells or offcells (Chalasani et al., 2007; Zimmer et al., 2009; this study). In thermotaxis, in contrast, initial indications are that the derivative is computed mainly at the cellular level, as the main thermosensory neuron class, AFD, exhibits a positive impulse to temperature upsteps and a negative impulse to temperature downsteps (Clark et al., 2006). This difference in computational strategy could be the result of differences in the cellular requirements for sensory transduction, or of differences in the behaviors associated with orientation to chemicals or temperature. Thus, a comparative analysis of the circuitry underlying chemotaxis, aerotaxis, and thermotaxis may provide new insights into the relationship between constraints operating at the cellular and behavioral levels.

\section{References}

Bargmann CI, Avery L (1995) Laser killing of cells in Caenorhabditis elegans. Methods Cell Biol 48:225-250.

Bargmann CI, Horvitz HR (1991) Chemosensory neurons with overlapping functions direct chemotaxis to multiple chemicals in C. elegans. Neuron 7:729-742.

Brenner S (1974) The genetics of Caenorhabditis elegans. Genetics 77:71-94. Chalasani SH, Chronis N, Tsunozaki M, Gray JM, Ramot D, Goodman MB, Bargmann CI (2007) Dissecting a circuit for olfactory behaviour in Caenorhabditis elegans. Nature 450:63-70.

Chalfie M, Sulston JE, White JG, Southgate E, Thomson JN, Brenner S (1985) The neural circuit for touch sensitivity in Caenorhabditis elegans. J Neurosci 5:956-964.

Clark DA, Biron D, Sengupta P, Samuel AD (2006) The AFD sensory neurons encode multiple functions underlying thermotactic behavior in $\mathrm{Cae}$ norhabditis elegans. J Neurosci 26:7444-7451.

Dusenbery DB (1980) Responses of the nematode Caenorhabditis elegans to controlled chemical stimulation. J Comp Physiol 136:127-331.

Gabel CV, Gabel H, Pavlichin D, Kao A, Clark DA, Samuel AD (2007) Neural circuits mediate electrosensory behavior in Caenorhabditis elegans. J Neurosci 27:7586-7596.

Hart AC, Sims S, Kaplan JM (1995) Synaptic code for sensory modalities revealed by C. elegans GLR-1 glutamate receptor. Nature 378:82-85.

Hart AC, Kass J, Shapiro JE, Kaplan JM (1999) Distinct signaling pathways mediate touch and osmosensory responses in a polymodal sensory neuron. J Neurosci 19:1952-1958.
Hilliard MA, Bargmann CI, Bazzicalupo P (2002) C. elegans responds to chemical repellents by integrating sensory inputs from the head and the tail. Curr Biol 12:730-734.

Hilliard MA, Apicella AJ, Kerr R, Suzuki H, Bazzicalupo P, Schafer WR (2005) In vivo imaging of C. elegans ASH neurons: cellular response and adaptation to chemical repellents. EMBO J 24:63-72.

Iino Y, Yoshida K (2009) Parallel use of two behavioral mechanisms for chemotaxis in Caenorhabditis elegans. J Neurosci 29:5370-5380.

Kaplan JM, Horvitz HR (1993) A dual mechanosensory and chemosensory neuron in Caenorhabditis elegans. Proc Natl Acad Sci U S A 90:2227-2231.

Kerr R, Lev-Ram V, Baird G, Vincent P, Tsien RY, Schafer WR (2000) Optical imaging of calcium transients in neurons and pharyngeal muscle of C. elegans. Neuron 26:583-594.

Mello C, Fire A (1995) DNA transformation. Methods Cell Biol 48:451-482.

Miller AC, Thiele TR, Faumont S, Moravec ML, Lockery SR (2005) Stepresponse analysis of chemotaxis in Caenorhabditis elegans. J Neurosci 25:3369-3378.

Nagai T, Yamada S, Tominaga T, Ichikawa M, Miyawaki A (2004) Expanded dynamic range of fluorescent indicators for $\mathrm{Ca}^{2+}$ by circularly permuted yellow fluorescent proteins. Proc Natl Acad Sci U SA 101:10554-10559.

Pierce-Shimomura JT, Morse TM, Lockery SR (1999) The fundamental role of pirouettes in Caenorhabditis elegans chemotaxis. J Neurosci 19:9557-9569.

Richmond JE, Davis WS, Jorgensen EM (1999) UNC-13 is required for synaptic vesicle fusion in C. elegans. Nat Neurosci 2:959-964.

Ryu WS, Samuel AD (2002) Thermotaxis in Caenorhabditis elegans analyzed by measuring responses to defined thermal stimuli. J Neurosci 22:5727-5733.

Sambongi Y, Nagae T, Liu Y, Yoshimizu T, Takeda K, Wada Y, Futai M (1999) Sensing of cadmium and copper ions by externally exposed ADL, ASE, and ASH neurons elicits avoidance response in Caenorhabditis elegans. Neuroreport 10:753-757.

Sambongi Y, Takeda K, Wakabayashi T, Ueda I, Wada Y, Futai M (2000) Caenorhabditis elegans senses protons through amphid chemosensory neurons: proton signals elicit avoidance behavior. Neuroreport 11:2229-2232.

Sarafi-Reinach TR, Melkman T, Hobert O, Sengupta P (2001) The lin-11 LIM homeobox gene specifies olfactory and chemosensory neuron fates in C. elegans. Development 128:3269-3281.

Schackwitz WS, Inoue T, Thomas JH (1996) Chemosensory neurons function in parallel to mediate a pheromone response in C. elegans. Neuron 17:719-728.

Suzuki H, Thiele TR, Faumont S, Ezcurra M, Lockery SR, Schafer WR (2008) Functional asymmetry in Caenorhabditis elegans taste neurons and its computational role in chemotaxis. Nature 454:114-117.

Sze JY, Victor M, Loer C, Shi Y, Ruvkun G (2000) Food and metabolic signalling defects in a Caenorhabditis elegans serotonin-synthesis mutant. Nature 403:560-564

Tobin D, Madsen D, Kahn-Kirby A, Peckol E, Moulder G, Barstead R, Maricq A, Bargmann C (2002) Combinatorial expression of TRPV channel proteins defines their sensory functions and subcellular localization in $C$. elegans neurons. Neuron 35:307-318.

Troemel ER, Chou JH, Dwyer ND, Colbert HA, Bargmann CI (1995) Divergent seven transmembrane receptors are candidate chemosensory receptors in C. elegans. Cell 83:207-218.

Ward S (1973) Chemotaxis by the nematode Caenorhabditis elegans: identification of attractants and analysis of the response by use of mutants. Proc Natl Acad Sci U S A 70:817-821.

White JG, Southgate E, Thomson JN, Brenner S (1986) The structure of the nervous system of the nematode Caenorhabditis elegans. Philos Trans R Soc Lond B Biol Sci 314:1-340.

Winer BJ, Brown DR, Michels KM (1991) Statistica principles in experimental design, Ed 3. Boston: McGraw-Hill.

Zariwala HA, Miller AC, Faumont S, Lockery SR (2003) Step response analysis of thermotaxis in Caenorhabditis elegans. J Neurosci 23:4369-4377.

Zheng Y, Brockie PJ, Mellem JE, Madsen DM, Maricq AV (1999) Neuronal control of locomotion in C. elegans is modified by a dominant mutation in the GLR-1 ionotropic glutamate receptor. Neuron 24:347-361.

Zimmer M, Gray JM, Pokala N, Chang AJ, Karow DS, Marletta MA, Hudson ML, Morton DB, Chronis N, Bargmann CI (2009) Neurons detect increases and decreases in oxygen levels using distinct guanylate cyclases. Neuron 61:865-879. 